\title{
Descripción de dos casos de síndrome de ALCAPA
}

\section{Description of two cases of ALCAPA syndrome}

\author{
Diana Vargas-Vergara1*, Fabián M. Heredia², Carlos A. Arias-Barrera ${ }^{1}$, Jeffrey Castellanos-Parada1, \\ Hugo A. Pabón-Enciso ${ }^{1}$ y Diana P. Otálora-Mancilla ${ }^{1}$ \\ ${ }^{1}$ Servicio de Cardiología; ${ }^{2}$ Servicio de Radiología. Clínica Universitaria Colombia, Bogotá Colombia
}

\begin{abstract}
Resumen
El origen anómalo de la arteria coronaria izquierda desde la arteria pulmonar o, por su sigla en inglés, síndrome de ALCAPA (Anomalous origin of the Left Coronary Artery from the Pulmonary Artery), también conocido como síndrome Bland-White-Garland es una rara cardiopatía congénita; sin embargo, es una de las causas más comunes de falla cardiaca, isquemia e infarto de miocardio en niños, que, en ausencia de tratamiento, alcanza una tasa de mortalidad del 35 al 85 \% en el primer año de vida. Hay dos tipos de síndrome de ALCAPA, aquel que se presenta en infantes y el que ocurre en adultos. El segundo tipo es raro y puede manifestarse como infarto de miocardio, disfunción ventricular izquierda e insuficiencia mitral, o isquemia silente que podría llevar a muerte súbita. Se presenta el caso de una paciente de 30 años, con cardiopatía dilatada y disfunción ventricular severa, secundaria a síndrome de ALCAPA, y el caso de un paciente de 61 años con cuadro de dolor torácico en quien se documentó el mismo síndrome.
\end{abstract}

Palabras clave: Síndrome de ALCAPA. Disfunción ventricular. Síndrome Bland-White-Garland.

\section{Abstract}

The anomalous origin of the left coronary artery from the pulmonary artery or its acronym ALCAPA syndrome (Anomalous Origin of the Left Coronary Artery from the Pulmonary Artery), also known as Blande-Whitee-Garland syndrome, is a rare congenital heart disease. However, one of the most common causes of heart failure, ischemia and myocardial infarction in children, which in the absence of treatment, reaches a mortality rate of 35 to $85 \%$ in the first year of life. There are two types of ALCAPA syndrome, that occurs in infants and adults. The presentation of the second type is rare and can manifest as myocardial infarction, left ventricular dysfunction and mitral insufficiency, or silent ischemia that could lead to sudden death. We present the case of a 30-year-old patient with dilated cardiomyopathy and severe ventricular dysfunction, secondary to the ALCAPA syndrome, another case of a 61-year-old patient with chest pain where the mentioned syndrome was documented.

Key words: ALCAPA syndrome. Ventricular dysfunction. Bland-White-Garland syndrome.

\section{Correspondencia:}

*Diana Vargas-Vergara E-mail: diani282@gmail.com licencia CC BY-NC-ND (http://creativecommons.org/licenses/by-nc-nd/4.0/).
Disponible en internet: 19-03-2021 Rev Colomb Cardiol. 2021;28(1):98-101 www.rccardiologia.com 


\section{Caso 1}

Mujer de 30 años, con cuadro de dos años de evolución, consistente en deterioro de la clase funcional hasta II/IV de acuerdo con la clasificación de la NYHA; edema en miembros inferiores, disnea paroxística nocturna y ortopnea, quien había consultado en múltiples ocasiones y había sido hospitalizada varias veces por dicha sintomatología, por lo cual era conocida en la institución donde consultó por primera vez por cuadro de dos meses consistente en agudización de los síntomas y deterioro de clase funcional hasta IV/IV de la NYHA. Ingresó a la unidad de cuidados intensivos por falla cardiaca, con perfil congestivo y de hipoperfusión, requerimiento de soporte inotrópico e inicio de terapia de reemplazo renal con hemodiálisis por síndrome cardiorrenal asociado.

Como antecedentes se diagnosticó síndrome de ALCAPA ocho meses antes de su ingreso e insuficiencia renal crónica estadio 5 (glomeruloesclerosis focal y segmentaria por resultado de biopsia del 16 de marzo de 2015), cardiopatía isquémica en fase dilatada con fracción de eyección del ventrículo izquierdo (FEVI) del $10 \%$, portadora de cardiodesfibrilador (CDI) como prevención primaria de muerte súbita desde abril de 2015, y en manejo con antagonistas de los receptores de angiotensina II, betabloqueador cardioselectivo, antagonistas de aldosterona y diurético de ASA.

Luego de compensar su estado clínico fue llevada a estudios de extensión para evaluar su condición cardiovascular. Se realizó nuevo ecocardiograma transtorácico que informó cardiopatía dilatada con hipoquinesia severa y compromiso severo de la función ventricular, fracción de eyección del $10 \%$, disfunción diastólica de tipo restrictivo, dilatación auricular izquierda severa, dilatación ventricular derecha severa con disfunción sistólica moderada a severa, insuficiencia tricúspide moderada, PSAP $55 \mathrm{~mm} \mathrm{Hg}$, dilatación severa de la arteria pulmonar, insuficiencia pulmonar moderada, electrodo con CDI en cavidades derechas.

Se realizó arteriografía coronaria izquierda, la cual mostró nacimiento anómalo de la arteria pulmonar en el segmento proximal. Al realizar inyección en la arteria coronaria derecha se apreció abundante circulación colateral que llenó todo el árbol coronario izquierdo, incluyendo el tronco, y se evidenció el nacimiento anómalo anotado, sin lesiones en su luz. Coronaria derecha dominante, dilatada, con nacimiento normal, sin lesiones obstructivas. Cateterismo derecho: aurícula derecha $25 \mathrm{~mm} \mathrm{Hg}$; ventrículo derecho 70/20 mm Hg; arteria pulmonar $71 / 20$ (39) $\mathrm{mm} \mathrm{Hg}$ y capilar pulmonar $30 \mathrm{~mm} \mathrm{Hg}$. Se complementaron imágenes de

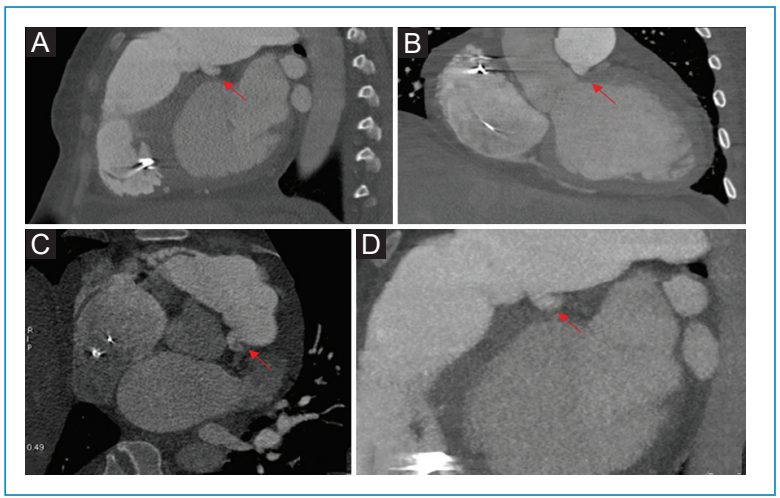

Figura 1. A: reconstrucción sagital oblicua de Angiotac gatillado que demuestra origen anómalo de la arteria coronaria izquierda desde el tronco principal de la arteria pulmonar (flecha roja). B: reconstrucción coronal de Angiotac demostrando origen anómalo desde la pared inferior del tronco principal de la arteria pulmonar (flecha roja). C: corte axial de angiotac gatillado demostrando origen anormal de la arteria coronaria principal izquierda y aumento del diámetro del tronco principal de la arteria pulmonar. D: reconstrucción sagital MIP demostrando origen anómalo de arteria coronaria principal (flecha roja) desde la pared inferior del tronco principal de la arteria pulmonar.

angiografía con angio-TAC de aorta torácica y de arterias coronarias para evaluar la posibilidad de tratamiento quirúrgico, la cual mostró síndrome de ALCAPA (Fig. 1), con origen de la arteria coronaria izquierda desde el margen inferior del tercio medio del tronco principal de la arteria pulmonar; cardiomegalia global; derrame pericárdico y fracción de eyección del ventrículo izquierdo del $24.1 \%$. Se evaluó viabilidad para evaluar beneficio de una intervención quirúrgica a través de perfusión miocárdica con estrés farmacológico, la cual reportó necrosis en territorio de la arteria descendente anterior, en extensión aproximada del $25 \%$ de la masa miocárdica, sin dilatación transitoria.

Con estos hallazgos se consideró que no se beneficiaba de la intervención quirúrgica de la anomalía coronaria, así que su tratamiento se encaminó a optimizar el manejo farmacológico y a evaluar la posibilidad de un trasplante dual corazón-riñón.

\section{Caso 2}

Paciente de 61 años, con cuadro de dolor torácico irradiado a cuello y maxilar, aproximadamente de 5 años de evolución, de carácter progresivo, con antecedentes de diabetes mellitus tipo 2, hipertensión arterial, dislipidemia e hipotiroidismo en tratamiento con losartán, ácido 


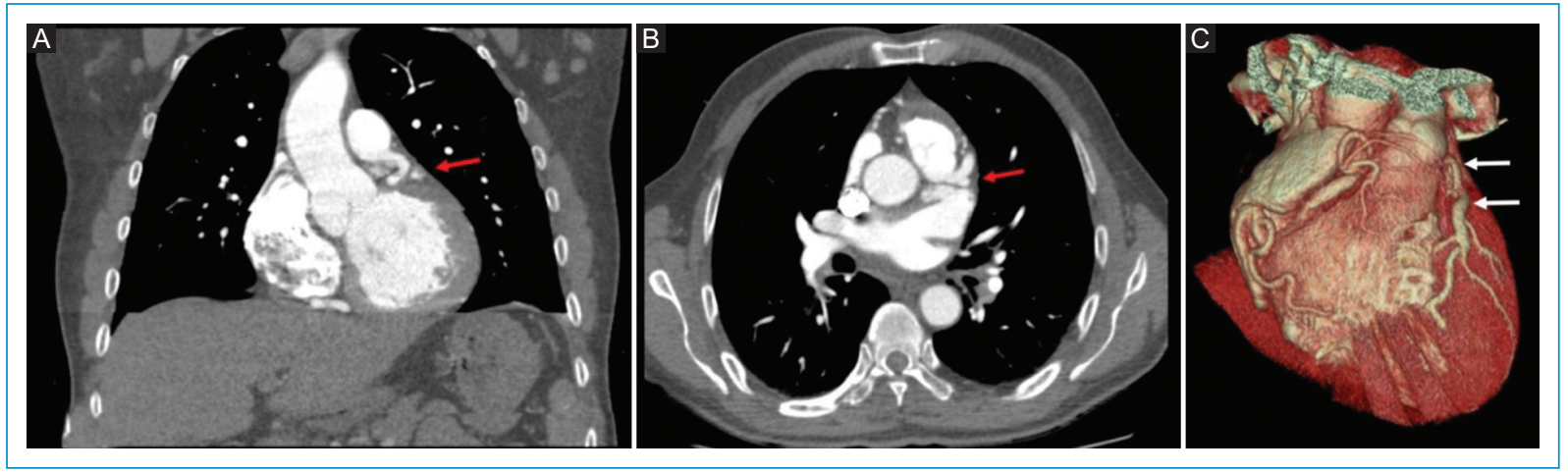

Figura 2. A: reconstrucción multiplanar coronal de Angiotac gatillado que muestra origen anómalo de la arteria coronaria principal izquierda a partir del tronco principal de la arteria pulmonar (flecha roja). B: corte axial de Angiotac que muestra origen anómalo de la arteria coronaria principal izquierda a partir de la arteria pulmonar principal (flecha roja). C: reconstruc ción volumétrica que demuestra arteria coronaria tortuosa y ectasia con origen en el tronco principal de la arteria pulmonar (flecha blanca).

acetil-salicílico, atorvastatina y levotiroxina. Se realizaron estudios con perfusión miocárdica, los cuales mostraron fracción de eyección del 41 \%, VFD 170 y VFS 100, positiva para isquemia anterior con extensión del $6 \%$. El ecocardiograma mostró cardiopatía dilatada, compromiso moderado de la función sistólica del ventrículo izquierdo del $40 \%$, disfunción diastólica tipo I, esclerosis valvular mitral y aórtica con insuficiencia mitral leve, y crecimiento auricular izquierdo. Se llevó a arteriografía coronaria en la que se evidenció nacimiento anómalo del tronco coronario izquierdo el cual emergía de la arteria pulmonar; además, arteria coronaria derecha severamente dilatada, con abundante llenado a la arteria coronaria izquierda, dilatación moderada del ventrículo izquierdo e hipertensión pulmonar. Posteriormente, se hizo angio-TAC de aorta torácica y de arterias coronarias (Fig. 2), en la que se confirmó anomalía de origen de la arteria coronaria izquierda, síndrome de ALCAPA (arteria coronaria izquierda: origen desde el tronco principal de la arteria pulmonar con un tronco común de aproximadamente $5 \mathrm{~mm}$ de longitud con diámetro de $6.5 \mathrm{~mm}$ ), circulación coronaria derecha dominante, importante tortuosidad, ectasia de la arteria coronaria derecha y fracción de eyección del ventrículo izquierdo del $58.3 \%$ ). Se documentó fibrilación auricular paroxística, por lo cual fue evaluado por cirugía cardiovascular, quienes realizaron injertos aorto-coronarios N2 de safena a la arteria descendente anterior y de safena a la obtusa marginal en "T", además de aislamiento de venas pulmonares más ligadura de la aurícula izquierda con atriclip off pump. El paciente tuvo una evolución adecuada posterior a la intervención. Se hizo seguimiento en controles por
Cardiología hasta completar un año posterior a la intervención, cuya evolución fue satisfactoria y resultó asintomático desde el punto de vista cardiovascular.

\section{Discusión}

El síndrome de ALCAPA, llamado así por sus siglas en inglés (Anomalous origin of the Left Coronary Artery from the Pulmonary Artery), es una rara cardiopatía congénita de la cual se entenderán dos tipos: el que se presenta en la infancia y el de la edad adulta ${ }^{1}$.

En la infancia es una de las causas más comunes de falla cardiaca, isquemia e infarto de miocardio². Su incidencia es de 1 por 300.000 nacidos vivos. La mortalidad en pacientes no tratados es del 35 al $85 \%$ en el primer año de vida ${ }^{3}$.

Ocasionalmente, los pacientes pueden sobrevivir hasta la edad adulta, cuando no han recibido tratamiento en la infancia; puede manifestarse como infarto de miocardio, disfunción ventricular izquierda e insuficiencia mitral o isquemia silente que podría llevar a muerte súbita ${ }^{2}$.

Durante la etapa fetal y neonatal temprana, la anomalía es bien tolerada debido a que la presión pulmonar es igual a la sistémica. Posteriormente, en la adaptación de la vida intrauterina a la extrauterina, la presión arterial pulmonar disminuye, al igual que el flujo en la arteria coronaria izquierda $(\mathrm{ACl})$, por lo que se presenta isquemia e infarto de miocardio. En el momento de la disminución del flujo de la $\mathrm{ACl}$ se desarrolla circulación colateral de la arteria coronaria derecha a la izquierda. Cuando está bien establecida se desarrolla el síndrome de ALCAPA del tipo de la edad 
adulta $^{3}$; sin embargo, el aporte al ventrículo izquierdo es insuficiente, en especial en la región subendocárdica, por lo que se produce isquemia crónica con disfunción ventricular, pudiendo estar inicialmente asintomático².

En el abordaje diagnóstico se han utilizado diferentes técnicas para su detección, algunas invasivas, como la arteriografía coronaria ${ }^{4}$, y otras no invasivas, como la TAC de arterias coronarias ${ }^{5,6}$, considerada esta última como de primera línea para el diagnóstico y seguimiento, ya que muestra de manera adecuada la anomalía y tiene el beneficio de aportar información adicional en caso de requerirse manejo quirúrgico ${ }^{2}$. Como parte de los estudios de extensión se han utilizado la RMN de corazón ${ }^{2}$ y la perfusión miocárdica ${ }^{5}$ para determinar la viabilidad miocárdica y así determinar si los pacientes pueden beneficiarse del tratamiento quirúrgico.

Los hallazgos imagenológicos primarios consisten en la visualización directa de la $\mathrm{ACl}$ saliendo de la cara antero-lateral izquierda de la arteria pulmonar; posteriormente, sigue el curso hacia el surco interventricular y se ramifica, formando las arterias descendente anterior y circunfleja; esta es la distribución usual. También puede observarse flujo retrógrado de la $\mathrm{ACl}$ a la arteria pulmonar en las imágenes de RMN de corazón, y jet de regurgitación por medio de SSFP cine (secuencia eco de gradiente en modo cine) $)^{2}$. Como hallazgos imagenológicos secundarios figuran: dilatación y tortuosidad de las arterias coronarias derecha e izquierda, evidencia de ramas colaterales dilatadas provenientes de la ACD (arteria coronaria derecha) a la $\mathrm{ACl}$, hipertrofia, dilatación y alteraciones de la contractilidad ventricular izquierda, degeneración mixomatosa y disfunción isquémica de los músculos papilares y adyacentes, la cual ocasiona insuficiencia y prolapso de la válvula mitral.

El realce tardío en RMN por isquemia subendocárdica crónica, hallazgo importante en pacientes asintomáticos o con diagnóstico incidental de la enfermedad, es un factor predictor de arritmias malignas, y en el seguimiento, si se observa empeoramiento de esta condición, debe evaluarse la posibilidad de tratamiento quirúrgico².

La corrección quirúrgica es considerada el tratamiento estándar, a menos que exista alguna contraindicación ${ }^{6}$. El método de elección es el reparo quirúrgico del defecto realizando redireccionamiento con parche o traslocación de la arteria coronaria izquierda anastomosándola en la aorta, con muy buenos resultados a largo plazo ${ }^{6}$, la cirugía de revascularización queda reservada para casos en que estas técnicas no son posibles y debe asociarse a ligadura del tronco de la coronaria. Otras opciones quirúrgicas incluyen trasplante cardiaco y cierre percutáneo de $\mathrm{ACl}$ anómala ${ }^{2,6}$.

\section{Financiamiento}

Los autores declaran que no tuvieron fuentes de financiamiento.

\section{Conflicto de intereses}

Los autores declaran que no existe conflicto de intereses.

\section{Responsabilidades éticas}

Protección de personas y animales. Los autores declaran que para esta investigación no se han realizado experimentos en seres humanos ni en animales.

Confidencialidad de los datos. Los autores declaran que han seguido los protocolos de su centro de trabajo sobre la publicación de datos de pacientes.

Derecho a la privacidad y consentimiento informado. Los autores han obtenido el consentimiento informado de los pacientes y/o sujetos referidos en el artículo. Este documento obra en poder del autor de correspondencia.

\section{Bibliografía}

1. Berhan Genc A, O"nder Dokso"z, Vedide Tavlı. A rare cause of dilated cardiomyopathy, the left coronary artery from the pulmonary artery (ALCAPA): Accurate diagnosis with dual-source CT. Journal of Indian College of Cardiology 2014;4:59-62.

2. Peña E, Nguyen ET, Merchant N, Dennie C. ALCAPA syndrome: No Just a Pediatric Disease. Radiographics. 2009;29(2):553-65.

3. Donataccio PM, Li W, Ramasamy M, Senior R. Anomalous origin of Left Coronary Artery from the Pulmonary Artery (ALCAPA): A rare presentation in late adulthood. Int J Cardiol. 2015;182:179-80.

4. Le Berre L, Fraisse A, Boulmierc D, Jiménez M, Mansourati J, Guerin P. Posters-Friday, 20 September 2013-12h00-13h00, Archives of Cardiovascular Disease. 2013;106:475-8.

5. Jiménez-Navarro MF, Alegre-Bayo AN, Algarra-García J. Diagnosis of ALCAPA syndrome in adults. Rev Esp Cardiol. 2009;62(10):1179.

6. Toumpourleka M, Belitsis G, Alonso R, Rubens M, Moat N, Gatzoulis M. Late presentation and surgical repair of ALCAPA. Int J Cardiol. 2015;186:207-9. 